\title{
Reoperative repair of adult aortic coarctation with explantation of thoracic stent-graft: a case report
}

\author{
N. Bryce Robinson ${ }^{1}$, Woodrow J. Farrington ${ }^{1}$, Peter Maresca ${ }^{2}$, Irbaz Hameed ${ }^{1}$, Erin M. Iannacone ${ }^{1}$, \\ Christopher Lau ${ }^{1}$, Mario Gaudino ${ }^{1}$, Leonard N. Girardi ${ }^{1}$ \\ ${ }^{1}$ Department of Cardiothoracic Surgery, Weill Cornell Medicine, New York, NY, USA; ${ }^{2}$ Department of Nursing, New York Presbyterian Hospital- \\ Weill Cornell Medical Center, New York, NY, USA \\ Correspondence to: Leonard N. Girardi, MD. Department of Cardiothoracic Surgery, Weill Cornell Medicine, 1300 York Avenue, New York, NY \\ 10065, USA. Email: lngirard@med.cornell.edu.
}

\begin{abstract}
Coarctation of the aorta $(\mathrm{CoA})$ is a relatively common congenital cardiac abnormality. Most patients undergo operative intervention early in life, and up to half of these patients will require reintervention at some point. Here we report our technique for operative repair of an adult coarctation in a patient previously treated with open bypass and endovascular stenting. The patient is a 50-year-old man with a history of hypertension and aortic coarctation treated with extra-anatomic bypass at age 16 who subsequently developed stenosis of the bypass with hypertension at age 45 and underwent endovascular stenting of the coarctation in 2013. He presented to our clinic with worsening hypertension and imaging showing a large pseudoaneurysm of the descending thoracic aorta (DTA). The patient underwent open explantation of the prior bypass and thoracic stent via left thoracotomy, with resection of the coarctation and reconstruction with a $22-\mathrm{mm}$ woven Dacron graft. Aorto-to-left-subclavian bypass was also performed with a $12-\mathrm{mm}$ Dacron graft. The patient was discharged on postoperative day 6, and at 2-month follow up remained in excellent functional status. This case represents a unique presentation of coarctation in a patient who had undergone two prior interventions. An open approach was deemed the most appropriate option given the patients' young age and failure of previous surgical measures and endovascular options. This case demonstrates that, when performed in a high-volume aortic center, explantation of a thoracic stent-graft with open repair of CoA can be safely performed with excellent outcomes.
\end{abstract}

Keywords: Reoperation; coarctation; cardiac surgery; endovascular; case report

Received: 15 April 2020; Accepted: 16 July 2020; Published: 20 January 2022.

doi: $10.21037 /$ jovs-20-106

View this article at: http://dx.doi.org/10.21037/jovs-20-106

\section{Introduction}

Coarctation of the aorta $(\mathrm{CoA})$ is a congenital heart abnormality that involves luminal narrowing of the aorta most commonly in close proximity to the ductus arteriosus, distal to the left subclavian artery. CoA is a relatively common abnormality, with a reported incidence of $6-8 \%$ in patients with congenital heart disease. It is associated with bicuspid aortic valve, which is present in up to half of patients, as well as intracranial aneurysms. Current ACC/ AHA guidelines recommend either surgical repair or catheter-based stenting for adults with hypertension and significant native or recurrent aortic coarctation defined as a resting gradient of $\geq 20 \mathrm{mmHg}$ (1). Other corrective strategies, such as endovascular balloon angioplasty, can be considered, but have higher rates of recurrence, aneurysm formation, and intimal tears (2). Following repair, patients are monitored frequently as they are at high risk of restenosis as well as aneurysm and pseudoaneurysm formation (3). In this case report, we demonstrate our surgical strategy for addressing recurrent $\mathrm{CoA}$ in a patient who had previously undergone extra-anatomic bypass and endovascular stenting. We present the following case in accordance with the CARE reporting checklist (available at https://jovs.amegroups.com/ 


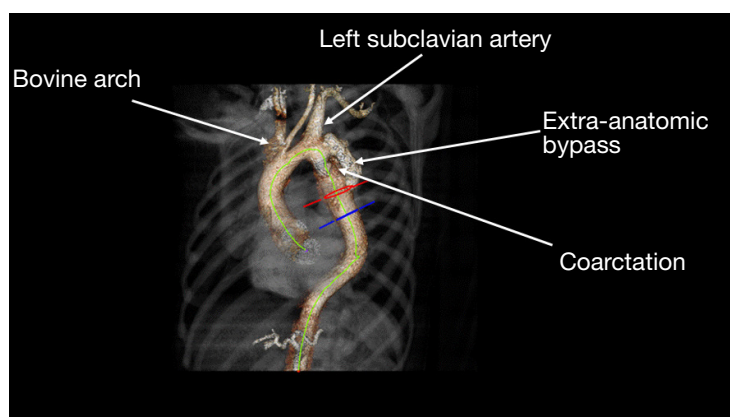

Figure 1 Preoperative CT-angiography showing extra-anatomic bypass with stented coarctation and dilated left subclavian artery. CT, computed tomography.

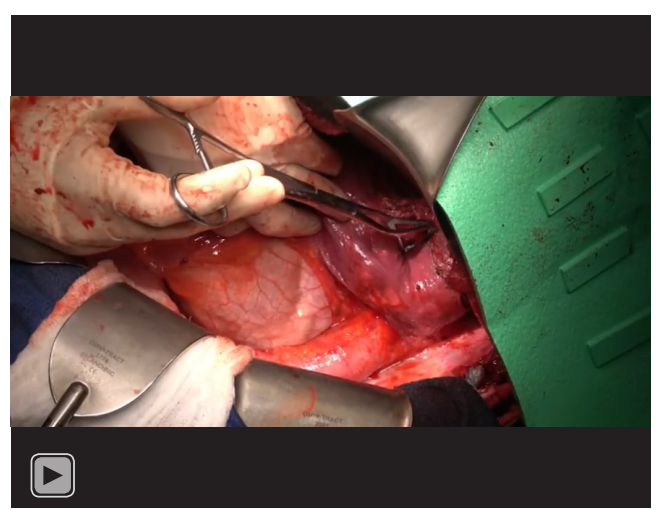

Video 1 Explantation of a thoracic stent-graft with open repair of coarctation of the aorta via left lateral thoracotomy.

article/view/10.21037/jovs-20-106/rc).

\section{Case presentation}

The patient is a 50 -year-old man with a history of hypertension and known intracranial aneurysms followed by Neurosurgery, who presented with a large pseudoaneurysm of the descending thoracic aorta (DTA) and recurrent coarctation. The patient underwent operative repair of an aortic coarctation at age 16 via left postero-lateral thoracotomy. The original bypass was performed in an extra-anatomic fashion around the coarctation from the proximal DTA to an area just distal to the coarctation. His recovery from the index operation was uneventful, and he did well until 2013 when he was found to have obstruction of the bypass graft. He subsequently underwent endovascular stenting of the original coarctation. Followup CT Angiogram performed in 2019 demonstrated a new disruption of the posterior intima of the bypass graft with contrast entering a focal outpouching medially with thrombus formation, suspicious for pseudoaneurysm formation (Figure 1). The DTA measured $5 \mathrm{~cm}$ in diameter. There was also mild dilation of the proximal left subclavian artery just distal to the takeoff measuring $2 \mathrm{~cm}$ in diameter. Due to the patient's history of hypertension, as well as the rapid expansion of the pseudoaneurysm, operative repair was recommended to avoid fatal rupture.

Preoperative workup included a transthoracic echocardiogram which demonstrated an ejection fraction of $60 \%$, with no regional wall motion abnormalities, trace mitral regurgitation, and a bicuspid aortic valve. Subsequent cardiac catheterization showed angiographically normal coronary arteries with mild aortic valve regurgitation. Pulmonary function testing revealed a FEV1 of $78 \%$ predicted and DLCO 101\% predicted. Room air arterial blood gas showed a $\mathrm{pH} 7.38, \mathrm{PaO}_{2} 112$, and $\mathrm{PaCO}_{2} 41$.

The patient was admitted to the hospital one day prior to the operation for preoperative cardiac catheterization, preoperative labs including urinalysis and culture, room air arterial blood gas, and intravenous hydration. He also was given a standard bowel preparation.

Equipment preference card: (I) $22 \mathrm{~mm}$ woven Dacron graft; (II) $12 \mathrm{~mm}$ woven Dacron graft; (III) 36 Fr chest tube $\times 2$; (IV) clip ligating horizon TI; (V) electric blade cautery extension (6.5 inches); (VI) retainer viscera (large and extralarge); (VII) softsilk 2-0 V-26 30 in; (VIII) prolene 2-0, 3-0, 4-0, 5-0; (IX) warming blanket.

The patient was brought to the operating room and placed in the supine position. General endotracheal anesthesia was induced without difficulty. A bronchial blocker was placed to allow for single-lung ventilation. A right radial arterial catheter, right internal jugular Swan-Ganz catheter, and foley catheter were placed for intraoperative monitoring and fluid/medication administration. A spinal drainage catheter was not used due to the presence of intracranial cerebral aneurysms.

The patient was then re-positioned in the right lateral decubitus position and prophylactic antibiotics were administered. The chest was entered through the previous thoracotomy, and the $5^{\text {th }}$ and $6^{\text {th }}$ ribs were shingled. Dense adhesions between the lung and chest wall were encountered (Video 1, Figure 2). Sharp dissection was used to expose the DTA beyond the bypass graft and distal control was obtained. Proximal control of the left subclavian artery was obtained high in the chest and proximal control of the aorta was obtained between the left carotid and 


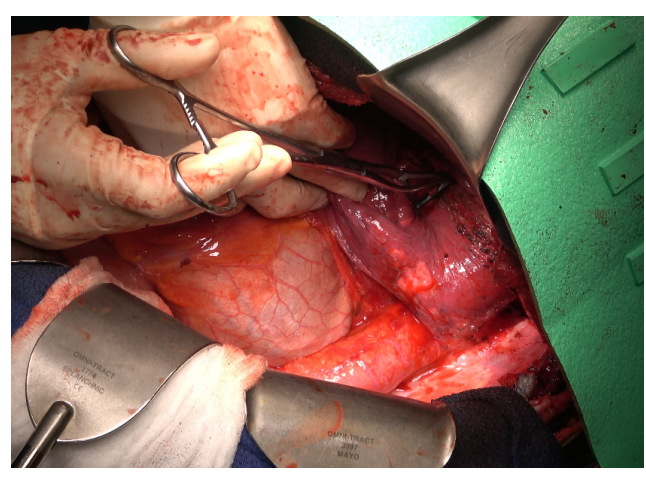

Figure 2 Exposure via left lateral thoracotomy. Dense adhesions are seen from the left lung to the distal anastomosis of the prior extra-anatomic bypass.
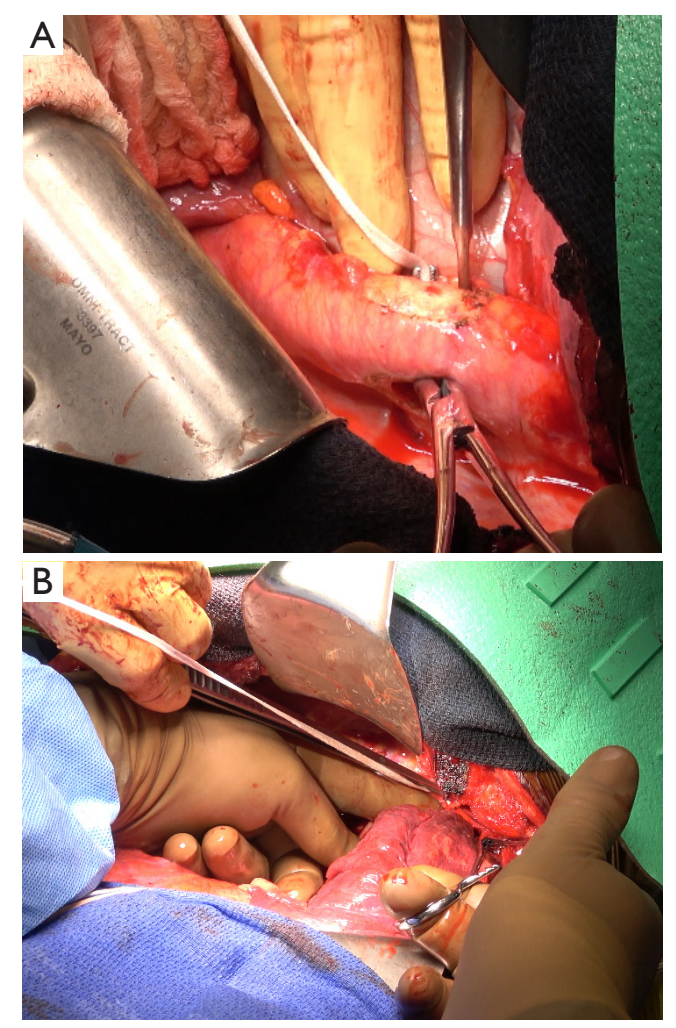

Figure 3 Control of the aorta is obtained. (A) Distal control of the aorta is obtained low in the chest; (B) proximal control of the left subclavian artery is obtained high in the chest between the left carotid and left subclavian arteries.

left subclavian arteries (Figure $3 A, 3 B$ ). The left vagus and phrenic nerves were identified. The vagus was adherent to the previous suture line and was divided.

Ten-thousand units of heparin were given and cross

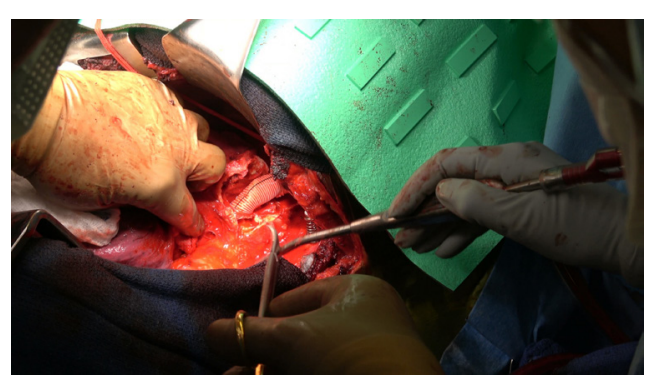

Figure 4 Prior extra-anatomic bypass graft is exposed and completely excised.

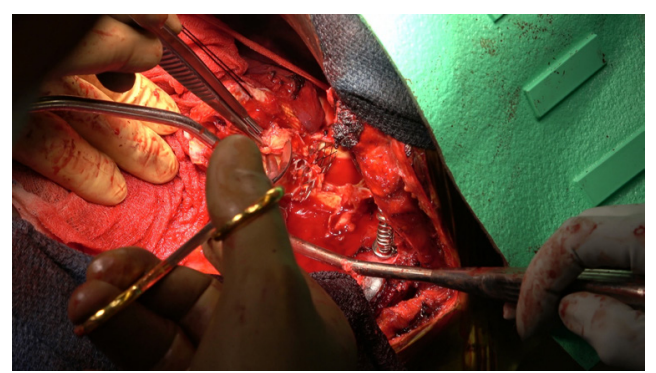

Figure 5 The native coarctation and metal stent are exposed and completely excised.

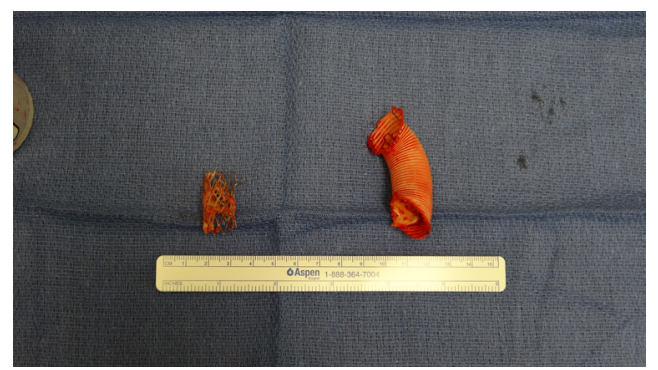

Figure 6 Left-excised bare metal stent. Right-excised extraanatomic bypass graft.

clamp applied to the aorta. A separate bull-dog clamp was applied to the subclavian artery, and a hypogastric clamp applied distally to the descending aorta low in the chest. The aorta was opened just beyond the distal suture line of the previous bypass graft and extended proximally to the origin of the left subclavian artery. The subclavian artery was divided off of the aneurysm sac. The previous coarctation shelf could be seen along with the stent graft, both of which were excised and removed (Figures 4-6).

The aorta was then dissected circumferentially off of 
the esophagus and trimmed back to normal tissue. Heavily back-bleeding intercostal arteries were oversewn with 2-0 silk suture. The aorta was reconstructed just distal the cross clamp beyond the left carotid artery using 2-0 prolene suture with a $22-\mathrm{mm}$ woven Dacron graft. The anastomosis was reinforced with pledgeted prolene sutures. The distal anastomosis was then carried out in a similar fashion. The cross clamp was then removed. Total cross clamp time was 33 minutes. The left subclavian artery was then reconstructed high in the chest in an end-to-end fashion using a $12-\mathrm{mm}$ woven Dacron graft and a running 5-0 prolene suture (Figure 7).

Protamine sulfate was then administered to reverse the heparin and hemostasis was achieved. Two chest tubes were placed in the left pleural cavity and the chest closed in the usual fashion.

The team consisted of one attending cardiothoracic surgeon, with a third-year cardiothoracic surgery fellow serving as first-assistant, and a trained cardiothoracic surgery physician assistant as second assistant. There

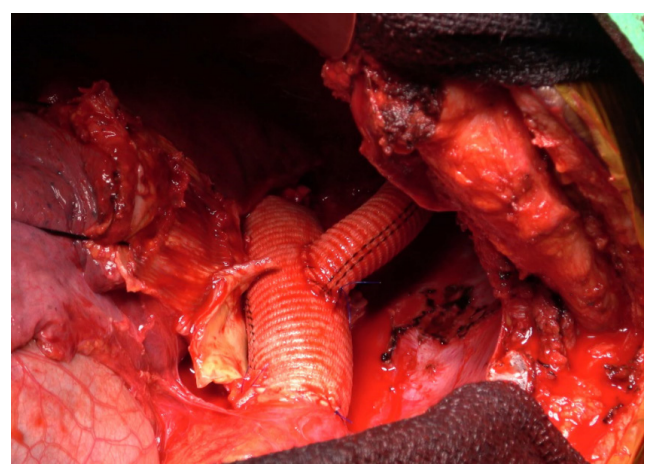

Figure 7 Completed anastomosis with left subclavian reconstruction. was one circulating nurse, and one bedside surgical technician. The anesthesia team consisted of an attending anesthesiologist and a cardiac-track anesthesia fellow, as well as two anesthesia residents and an anesthesia assistant.

The patient was brought to the intensive care unit intubated on minimal inotropic support. Our standard approach to managing DTAs and thoracoabdominal aneurysms (TAAA) alike include strict blood pressure management with mean arterial pressure greater than $85 \mathrm{mmHg}$, hemoglobin greater than 10, and cerebrospinal fluid (CSF) drainage to maintain an ICP of $12 \mathrm{mmHg}$ or less. Typically, CoA are managed no differently and follow the same course of management; however, given this patient's history of cerebral aneurysms a CSF drain was foregone. Should a CSF drainage catheter be used, it is imperative to monitor the patient's physical exam, hourly drainage volume, and the character of the fluid in the drainage reservoir. When present, it is our practice to clamp the CSF drain on the morning of postoperative day 2 and remove the drain if the neurological exam remains unchanged. Although a rich collateral network supplying the spinal cord exists in patients with CoA, the robustness of the networks is difficult to objectively quantify; therefore, we maintain the blood pressure goals similarly to DTAs and TAAAs.

The patient was extubated on the first postoperative day, and discharged home on postoperative day 6 . At 2-week follow up, the patient complained of a hoarse voice and was subsequently diagnosed with left vocal cord paralysis. Otolaryngology referral was made, and the patient underwent injection laryngoplasty under magnified digital laryngoscopic guidance with improvement both subjectively and on laryngeal EMG. At 8-week follow up the patient reported excellent functional status. A full timeline of events is available in Figure 8.

All procedures performed in this study were in accordance

Timeline of Events

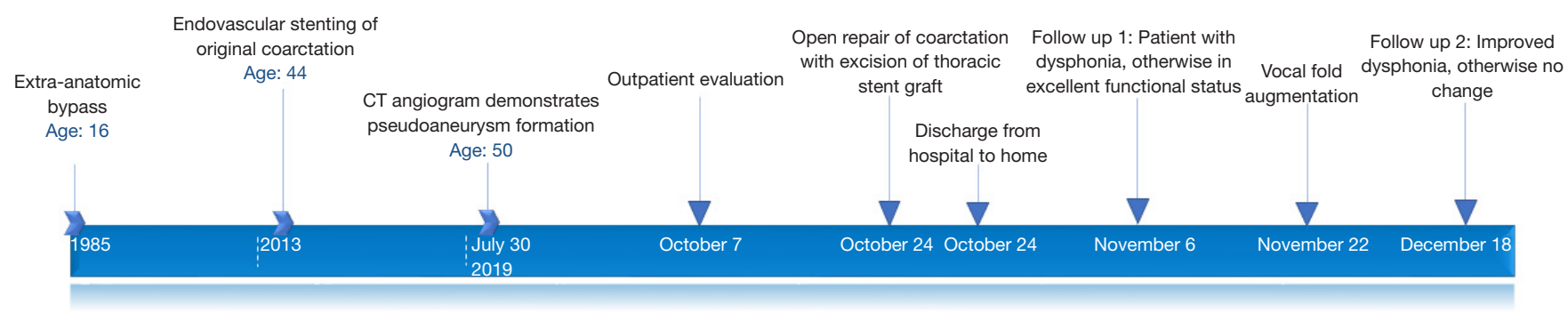

Figure 8 Detailed timeline or pre-, intra-, and post-operative events. 
with the ethical standards of the institutional and/or national research committee(s) and with the Helsinki Declaration (as revised in 2013). Written informed consent was obtained from the patient for publication of this case report and any accompanying images.

\section{Discussion}

CoA presents a unique set of technical challenges. Chief amongst these is selection of the appropriate correction strategy, especially in patients who present with recoarctation or aneurysm. Current US and European guidelines recommend an endovascular approach as the first strategy in these patients $(1,4)$.

In the modern era, open surgical correction is often shunned by the novelty of a less invasive approach; however, its purpose remains broad with appropriately selected patients. While the periprocedural risk associated with endovascular stenting for recurrent coarctation in adults is lower than that of open surgery, the long-term reintervention rate is of significant consideration. With the notable exception of the Coarctation of the Aorta Stent Trial (COAST), the evidence regarding reintervention rates is mostly limited to retro- and prospective singlecenter series. In published series with follow-up extending beyond 1 year, reported rates of reintervention associated with endovascular stenting range from $10 \%$ to $20 \%(5-7)$. In a review of the literature published between 1995 and 2005 , Carr and others reported a pooled restenosis rate of $11 \%$ in patients undergoing stent therapy, compared to $2 \%$ in those who underwent surgery (8). Our goal is to afford the patient freedom from reintervention while limiting the perioperative risks.

A complete preoperative assessment including contrasted computed tomography (CT), coronary anatomy, cardiac valve function, pulmonary function test, and baseline arterial blood gas plays an essential role in selecting the treatment modality and determining any cardiac adjuncts to be used during the operation. This clinical scenario provided a patient with a history of surgical correction of a coarctation at age 16 by way of an ascending to descending extra anatomic aortic bypass. This repair functioned adequately until age 50 at which time the patient developed evidence of stenosis and thrombosis of the bypass. An endovascular stent was placed in the native aorta without adequate relief of the pressure gradient across the coarctation. Additionally, development of a pseudoaneurysm of the distal aortic suture line followed the stent placement. Given the patient's young age and failure of previous surgical measures and endovascular options, our surgical plan included a posterolateral thoracotomy with resection of the prior extra anatomic bypass and stent followed by reconstruction of the descending aorta with a Dacron tube graft via the clamp and sew technique.

Specific attention should be directed to the CT imaging to determine an ideal proximal aortic clamp site for adequate resection and reconstruction. It is common for these patients to have some element of a hypoplastic arch that may complicate the proximal anastomosis or necessitate full cardiopulmonary bypass with deep hypothermic cerebral protection. Echocardiographic characteristics of the aortic valve and ascending aorta should not be ignored given the close association of CoA, bicuspid aortic valve disease and aortic aneurysmal disease. Patients with poor pulmonary reserve as indicated by preoperative testing can identify a specific patient population in which endovascular treatment may limit perioperative morbidity and mortality. Another important consideration was that this patient never had his original coarctation resected. Prior studies have shown that remnant ductal tissue, which is prone to fibrosis and subsequent constriction, is significantly associated with re-coarctation (9).

Operative monitoring and positioning include placement of an arterial cannula in the right upper extremity for monitoring while the aorta is clamped; additionally, an internal jugular PA catheter and a right femoral venous catheter are placed for hemodynamic monitoring and intravascular access. A spinal drain is placed in all patients and a strict postoperative protocol dictating hourly drainage and ICP goals is used. The patient is positioned in the right lateral decubitus position with the hips externally rotated to 45 degrees for access to the left femoral vessels if needed.

For this case the thoracic cavity is entered through the fifth intercostal space with division of the sixth rib posteriorly and costal cartilage anteriorly. The inferior pulmonary ligament is divided allowing unobstructed access to the distal thoracic aorta and left inferior pulmonary vein for drainage should partial cardiopulmonary bypass be utilized. The pleural covering is then opened overlying the distal aortic arch. The vagus and left recurrent laryngeal nerve are then identified and mobilized anteromedially. Typically, the area of coarctation is located just distal to the left subclavian artery within close proximity to the insertion of the ligamentum arteriosum. As such, division of the ligamentum arteriosum is necessary to safely achieve proximal control of the aorta between 
the carotid and subclavian artery and augment resection of all abnormal tissue as well as to facilitate sewing of the proximal anastomosis. Circumferential dissection of the subclavian artery is obtained in preparation for reimplantation following completion of the proximal aortic anastomosis. Once the proximal aortic clamp is placed, the aorta is opened longitudinally and the intercostal arteries are oversewn with 2-0 silk suture. When transecting the proximal aorta care must be taken to identify and protect the esophagus just below the transection plane. It is our practice to complete the proximal anastomosis using a circumferential running 3-0 prolene suture. The proximal anastomosis is then reinforced with sequential 3-0 pledgeted prolene sutures, starting on the posterior suture line. The cross clamp is then moved to a level below the anastomosis and hemostasis is confirmed. The distal anastomosis is then carried out in a likewise manner. Since the segment of aortic repair was short, intercostal artery reimplantation was not performed. In our practice, this is generally reserved for aortic replacement extending below the level of T10-T12. In addition to confirming adequate hemostasis, it is good practice to survey the operative field around the proximal anastomosis for lymphatic fluid as the thoracic duct may have an abnormal course and can be disrupted at this level.

For this case, we elected to use a clamp and sew technique. Though subject to scrutiny, we continue to maintain this method in approximately $65 \%$ of our DTAs and TAAAs with excellent results if an expeditious repair is anticipated. Given the rich collateral network supplying the spinal cord in patients with CoA, we have not felt the need to use partial bypass universally in this patient population. Similarly, the Coselli group has published similar data with a clamp and sew technique in a majority of patients with excellent results (10). To be sure, the specific therapy should be a customized to each patient and a decision that is carefully weighed by each individual surgeon against preoperative comorbidities, anatomic, and lifestyle considerations. To conclude, Explantation of a thoracic stent-graft with open repair of CoA can be safely performed in a high-volume aortic center.

\section{Acknowledgments}

Funding: None.

\section{Footnote}

Provenance and Peer Review: This article was commissioned by the Guest Editors (Roberto Di Bartolomeo, Davide Pacini and Mohamad Bashir) for the series "Best Video Presentation Prize for the 10th Postgraduate Course on 'Surgery of the Thoracic Aorta' in Bologna" published in Journal of Visualized Surgery. The article has undergone external peer review.

Reporting Checklist: The authors have completed the CARE reporting checklist. Available at https://jovs.amegroups. com/article/view/10.21037/jovs-20-106/rc

Conflicts of Interest: All authors have completed the ICMJE uniform disclosure form (available at https://jovs.amegroups. com/article/view/10.21037/jovs-20-106/coif). The series "Best Video Presentation Prize for the 10th Postgraduate Course on 'Surgery of the Thoracic Aorta' in Bologna" was commissioned by the editorial office without any funding or sponsorship. LNG serves as an unpaid editorial board member of Fournal of Visualized Surgery from July 2018 to June 2022. The authors have no other conflicts of interest to declare.

Ethical Statement: The authors are accountable for all aspects of the work in ensuring that questions related to the accuracy or integrity of any part of the work are appropriately investigated and resolved. The Institutional Review Board at Weill Cornell Medical Center waived the need for approval. All procedures performed in this study were in accordance with the ethical standards of the institutional and/or national research committee(s) and with the Helsinki Declaration (as revised in 2013). Written informed consent was obtained from the patient for publication of this case report and accompanying images/ video. A copy of the written consent is available for review by the editorial office of this journal.

Open Access Statement: This is an Open Access article distributed in accordance with the Creative Commons Attribution-NonCommercial-NoDerivs 4.0 International License (CC BY-NC-ND 4.0), which permits the noncommercial replication and distribution of the article with the strict proviso that no changes or edits are made and the original work is properly cited (including links to both the formal publication through the relevant DOI and the license). See: https://creativecommons.org/licenses/by-nc-nd/4.0/.

\section{References}

1. Stout KK, Daniels CJ, Aboulhosn JA, et al. 2018 AHA/ACC 
Guideline for the management of adults with congenital heart disease: a report of the American College of Cardiology/ American Heart Association Task Force on Clinical Practice Guidelines. Circulation 2019;139:e698-800.

2. Beckmann E, Jassar AS. Coarctation repair-redo challenges in the adults: what to do? J Vis Surg 2018;4:76.

3. Brown ML, Burkhart HM, Connolly HM, et al. Late outcomes of reintervention on the descending aorta after repair of aortic coarctation. Circulation 2010;122:S81-4.

4. Erbel R, Aboyans V, Boileau C, et al. Corrigendum to: 2014 ESC Guidelines on the diagnosis and treatment of aortic diseases. Eur Heart J 2015;36:2779.

5. Meadows J, Minahan M, McElhinney DB, et al. Intermediate outcomes in the prospective, multicenter Coarctation of the Aorta Stent Trial (COAST). Circulation 2015;131:1656-64.

doi: 10.21037/jovs-20-106

Cite this article as: Robinson NB, Farrington WJ, Maresca $\mathrm{P}$, Hameed I, Iannacone EM, Lau C, Gaudino M, Girardi LN. Reoperative repair of adult aortic coarctation with explantation of thoracic stent-graft: a case report. J Vis Surg 2022;8:8.
6. Erben Y, Oderich GS, Verhagen HJM, et al. Multicenter experience with endovascular treatment of aortic coarctation in adults. J Vasc Surg 2019;69:671-9.e1.

7. Moltzer E, Roos-Hesselink JW, Yap SC, et al. Endovascular stenting for aortic (re)coarctation in adults. Neth Heart J 2010;18:430-6.

8. Carr JA. The results of catheter-based therapy compared with surgical repair of adult aortic coarctation. J Am Coll Cardiol 2006;47:1101-7.

9. Jonas RA. Coarctation: do we need to resect ductal tissue? Ann Thorac Surg 1991;52:604-7.

10. Coselli JS, LeMaire SA, Conklin LD, et al. Left heart bypass during descending thoracic aortic aneurysm repair does not reduce the incidence of paraplegia. Ann Thorac Surg. 2004;77:1298-303; discussion 1303. 